EMBRYARIDDLE
Aeronautical University

SCHOLARLY COMMONS

\section{International Journal of Aviation,} Aeronautics, and Aerospace

$10-1-2017$

\title{
Identification of Reverse Engineering Candidates utilizing Machine Learning and Aircraft Cannibalization Data
}

\author{
Marc Banghart \\ Embry Riddle Aeronautical University, bangh14b@erau.edu
}

Follow this and additional works at: https://commons.erau.edu/ijaaa

Part of the Industrial Engineering Commons, Management Sciences and Quantitative Methods Commons, Operational Research Commons, Operations and Supply Chain Management Commons, Other Operations Research, Systems Engineering and Industrial Engineering Commons, Risk Analysis Commons, and the Systems Engineering Commons

\section{Scholarly Commons Citation}

Banghart, M. (2017). Identification of Reverse Engineering Candidates utilizing Machine Learning and Aircraft Cannibalization Data. International Journal of Aviation, Aeronautics, and Aerospace, 4(4). https://doi.org/10.15394/ijaaa.2017.1183

This Article is brought to you for free and open access by the Journals at Scholarly Commons. It has been accepted for inclusion in International Journal of Aviation, Aeronautics, and Aerospace by an authorized administrator of Scholarly Commons. For more information, please contact commons@erau.edu. 
Aircraft in the United States military continue to age and remain in service far exceeding their original design life. In addition to the accumulated age of the aircraft (and their associated systems and components), budget cuts have placed additional strain on the associated aircraft supply chains and maintainers.

Cannibalization, where a donor aircraft is utilized to provide spare parts for another, is commonly utilized to maintain readiness when spares are not available. The failed aircraft is immediately fixed, and a spare is backordered for the donor aircraft. The strategy has become prevalent and considered a "routine aircraft maintenance strategy" by the Government Accountability Office (GAO). The GAO has estimated that the Navy and Air Force executed 154-176,000 cannibalizations per year (FY 1996 - 2000) costing over 5 million maintenance man-hours (Government Accountability Office, 2001). Cannibalization of parts does allow repair of an aircraft however; it also demonstrates an underlying failure of the supply system. Additionally, it introduces additional maintenance requirements and may impact morale (Albright, Gerber, \& Juras, 2014). Although cannibalization has several drawbacks, the strategy can be seen as viable if supported by analysis of results (Enslen, 2004).

According to recent reports the practice of cannibalization is occurring frequently and has not diminished since the 2001 GAO study. For example, the submarine force cannibalizes at the rate of 1.5 components per day. Additionally, delays have resulted in submarines remaining for far longer in dry-dock (Seck, 2016). The Air Force is experiencing similar problems, with increasing number of parts having to be sourced from retired aircraft (Griffin \& Tomlinson, 2016).

Spare shortages resulting in the need for cannibalization can be managed utilizing several strategies. First, if the shortages are limited to certain geographic locations then the supply chain agility should be improved. Perhaps if an overseas location typically utilizes a higher proportion of hydraulic actuators than other locations, spare parts can be prepositioned at or near this location. In many cases the shortage of spare parts is systemic and not limited to one or two geographic locations. In these situations, several other strategies may be used. These solutions range from modification of operational loads or associated repair programs, improvement of part reliability, or re-opening of manufacturing lines. In many cases, reverse engineering is utilized in order to qualify new vendors or locally manufacture spares.

The trend of reverse engineering is likely to continue due to improvements in manufacturing capability, specifically the advent of additive manufacturing (or $3 \mathrm{D}$ printing). In order to reverse engineer a product, several steps must be followed. First, the geometry of the component must be established and a Computer Aided Drawing (CAD) model is developed. For complex parts that include electrical, 
pneumatic, or hydraulic design features, the various signals and theory of operation must be fully described. Once the CAD model has been developed to an acceptable level of fidelity, the production phase is entered. The design and reverse engineering life cycles are provided in Figure 1 for reference.

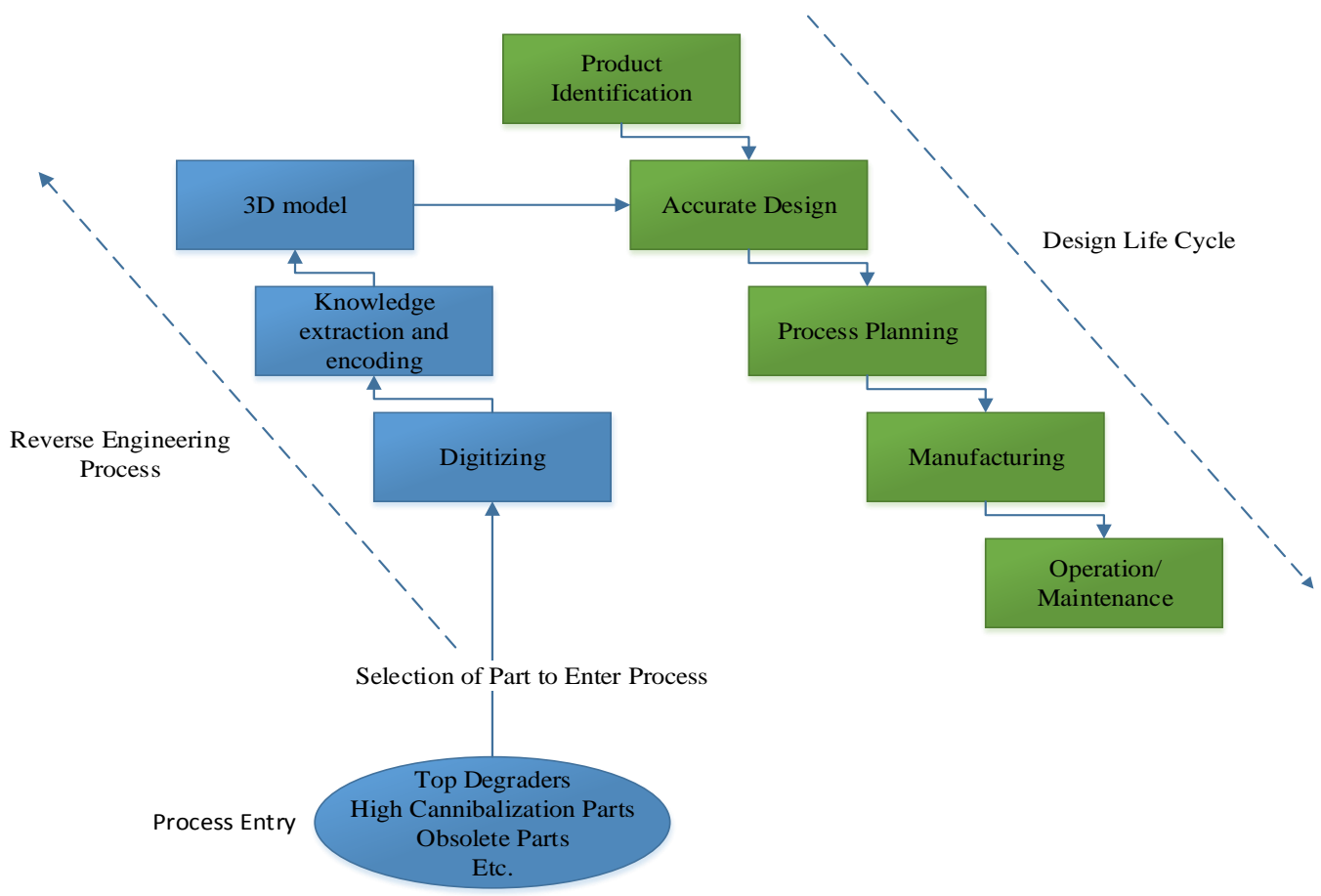

Figure 1. Interaction of the Reverse Engineering Process and Part Design Life Cycle adapted from (Durupt, Sebastien, Ducellier, \& Bricogne, 2010)

The efficient and proactive identification of parts and components to enter the reverse engineering process is critical to maintain readiness. Additionally, reverse engineering of parts that can reduce the number of cannibalization actions will reduce overall life cycle cost. Furthermore, the reverse engineering process can take a significant amount of time to accomplish; thus analytical methods that can support proactive identification of candidates are important.

Methods that proactively identify parts that may require cannibalization to ensure readiness would provide significant benefits to decision makers in several ways. Proactively understanding the likelihood of cannibalization actions can inform several engineering processes. The associated supply chain can be improved, reverse engineering activities can be undertaken, or additional manufacturing sources can be identified. The advancement of technologies such as 
additive manufacturing, coupled with machine learning may further yield positive results. Of course, several additional challenges must be overcome to implement additive manufacturing. These include, but are not limited to, understanding the material properties of printed parts, integration of parts in larger complex systems, and flight certification of the printed component.

This contribution applies a machine learning algorithm to a real-world military maintenance data set in order to develop a predictive methodology focused on improving decision making considering cannibalization actions. The chosen data set is representative of any typical military or civilian aircraft, and the developed methodology can be applied to any aircraft by selecting the appropriate training data set. The contribution fills an important gap within current literature, provides a methodology, and identifies a causal network that can inform decision making to enable quantification of cannibalization risk.

\section{Literature Review}

Literature that identifies an optimum cannibalization policy or top degraders at risk of impending cannibalization is sparse (Zhang \& Ghanmi, 2014). Several early papers were published (Rolfe, 1970; Fisher, 1986; Fisher 1989) during the 1970-1980s, formulating the underlying mathematical theory of cannibalization. These papers, along with subsequent contributions, focus on the determination of optimal policies with varying constraints or simplifying assumptions. The theory was expanded by Cheung and Hausman (1995) as well as Sung and Kim (2001). A simulation based method has also been developed by Salman, Cassady, Pohl, and Ormon (2007). Salman et al. proposed a decision making tool implemented using discrete-event simulation in order to assess the impact of cannibalization in terms of cost and readiness. They considered damage induced during maintenance as well as limited spares. Salman et al. acknowledged that further research was needed to ascertain the appropriate reliability, maintainability, and cost parameters utilized in their model and that causal linkages between cannibalization and other maintenance related parameters must be explored.

More recently, Zhang and Ghanmi (2014) expanded upon previous research and developed a stochastic model implemented at the operational level. In their formulation, they considered several operational decisions, to include cannibalization, installation, repair, and replacement. Their method assumed that the underlying system is closed in nature (thus no spares enter/exit system) and that the total number of end items remain constant. The underlying assumption will not remain valid in most military applications where aircraft may be lost due to conflict, and additional spares may enter the system due to new purchase orders or reverse engineering activities. 
Okyere-Boateng (2015) performed a detailed statistical analysis of U.S. Navy cannibalization data and identified reasons driving cannibalization, which included supply shortages, high operational tempo, high readiness demands, and the supply response time. The research included the identification of the top cannibalized components over several years, and indicated that the associated list of components varied significantly, further complicating traditional forecasting strategies. Although the study provided several recommendations for improvement of the cannibalization process, no quantitative thresholds were derived in order to provide decision makers feedback as to the likelihood of a cannibalization action occurring. Additionally, the literature currently does not cover the application of advanced predictive analytics algorithms in order to forecast the probability of a cannibalization action.

Predictive maintenance has been studied in the literature. The goal of predictive maintenance is to determine an optimal maintenance schedule based on cost and equipment status. Thus, the goal is to perform maintenance at the optimum cycle. Application of machine learning algorithms to these types of problems has been discussed in the literature. Liu and Shen (2015) developed a neural networkbased model incorporating the concept of real-time updates assuming connection to a Big Data platform. Li, Chen, and Zhu (2016) also considered utilizing a Bayesian Network coupled with real-time updates to assess the risk of failure in the oil and gas industry and mitigation strategies. However, the research has not examined cannibalization coupled with machine learning.

\section{Method}

Bayesian prediction allows the calculation of the probability of an event that a target feature assumes a specified level, given the state of a set of descriptive features. Thus, the generalized Bayes' Theorem can be stated as (Kellerher, Mac Namee, \& D'Arcy, 2015)

$$
P(t=l) \mid q[1], \ldots q[m])=\frac{P(q[1], \ldots q[m] \mid t=l) P(t=l)}{P(q[1], \ldots, q[m])}
$$

where

$$
\begin{aligned}
& t \text { denotes the target feature, } \\
& \text { ldenotes the specified level, and } \\
& q \text { refers to the descriptive features }
\end{aligned}
$$

Bayesian networks allow encoding of causality between various descriptive features given a data set. The network can both be utilized for forward and backward prediction, as well as diagnostic functions (Li, Chen, \& Zhu, 2016). The network consists of nodes and edges, where each node represents a single feature. 
Edges represent the probabilistic (or causal) relationships between features. Each node also includes a conditional probability table which represents the probability distribution of the node feature conditioned on the connected descriptive features (Kellerher, Mac Namee, \& D'Arcy, 2015). Bayesian networks can be updated in real-time as additional evidence or information becomes available, allowing for statistical inference and reasoning under uncertainty. The update capability of Bayesian networks has resulted in application of the method in several domains to include dynamic risk assessment in the oil and gas industry $(\mathrm{Li}, \mathrm{Chen}, \& \mathrm{Zhu}$, 2016). Bayesian networks have also been applied in the medical community to investigate causality within cancer processes (Kaiser, Bland, \& Klinke II, 2016).

The causal structure of the Bayesian network can be estimated utilizing domain expertise or machine learning algorithms. One algorithm that has been shown to work well in this domain is tabu search. The reader is referred to Zargoush, Alemi, Vinzi, Vang, \& Kheirbek (2014) for a comparison of various different structure learning algorithms. Several local search scoring functions can be utilized in optimization-based local search methods to include the Bayesian information criterion (BIC). The tabu search algorithm extends the hill climber algorithm by the introduction of a tabu list. Specifically, the tabu list are the $n$ most recent moves. Once a local optimum is found, the algorithm steps to the least worst candidate in the immediate neighbor as long as the candidate is not within the tabu list (Margaritis, 2003).

As illustrated in Figure 2, an initial network structure is initialized. Frequently, the initial network structure takes the form of a Naïve Bayes network where conditional independence is assumed between the descriptive features. During each iteration a local score is calculated for the network. In iteration 2, an arc is deleted between $\mathrm{A}$ and $\mathrm{C}$, followed by recalculation of the local score metric. As illustrated in the example, the algorithm reverts back to the network structure developed in iteration 1. An arc is added from $\mathrm{C}$ to $\mathrm{E}$, resulting in an improved score. The process continues until the stopping criteria is reached. Additionally, moves such as reversing an arc are also allowed.

Several different score metrics can be utilized, including the Bayesian Dirichlet (with several variations), minimum description length or Bayesian Information Criterion, and the Akaike Information Criterion. Yang, Chang, and K. (2002) compared several different metrics in order to ascertain which performed optimally. The reader is referred to their work for a more detailed discussion of the various metrics, their assumptions, and performance. 

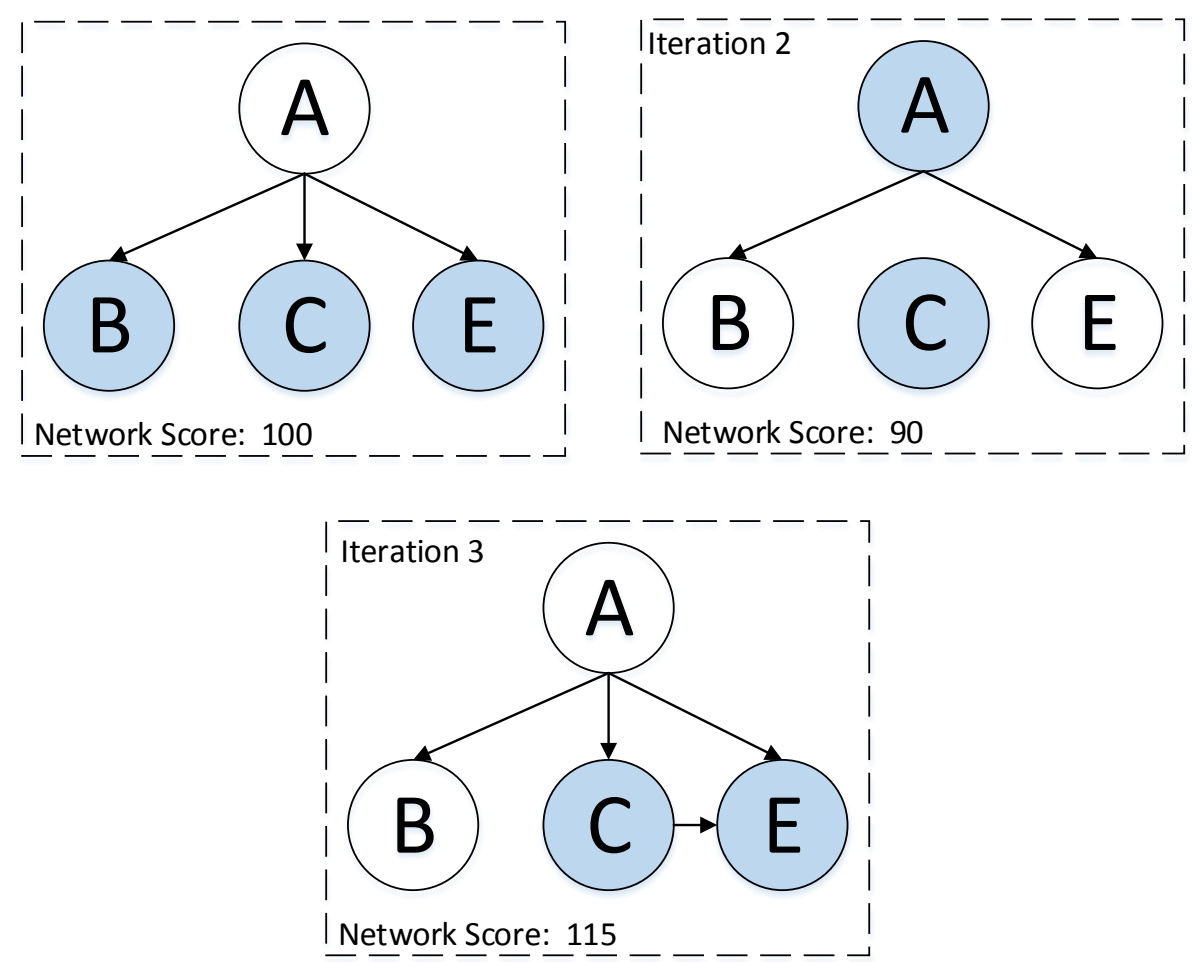

Figure 2. Illustration of Bayesian Network Construction using Machine Learning

The Bayesian local score metric was used along with a 10 fold crossvalidations scheme. First, we defined the number of records in our dataset, $D$ as $N$ with an associated network structure $B_{s}$. We further defined the cardinality of a feature, $x_{i}$ as $r_{i}$ where $1 \leq i \leq n$. Next, we defined the cardinality of a parent set of $x_{i}$ within our network structure as $q_{i}=\prod_{x_{j} \in p\left(x_{i}\right)} r_{j}$. The number of records in our dataset for which the probability of $x_{i}$ assumed it is the $j$ th value is denoted by $N_{i j}\left(1 \leq i \leq n, 1 \leq j \leq q_{i}, 1 \leq k \leq r_{i}\right)$. We denote $N_{i j}=\sum_{k=1}^{r_{i}} N_{i j k}$ and let $P\left(B_{S}\right)$ represent a prior on the network structure. Finally, we defined the Bayesian metric as (Bouckaert, 1995):

$$
Q\left(B_{s}, D\right)=P\left(B_{S}\right) \prod_{i=0}^{n} \prod_{j=1}^{q_{i}} \frac{\Gamma\left(\mathrm{N}_{i j}^{\prime}\right)}{\Gamma\left(\mathrm{N}_{i j}^{\prime}+N_{i j}\right)} \prod_{k=1}^{r_{i}} \frac{\Gamma\left(\mathrm{N}_{i j k}^{\prime}+N_{i j k}\right)}{\Gamma\left(\mathrm{N}_{i j k}^{\prime}\right)}
$$




\section{Case Analysis}

\section{Introduction}

Military organizations collect large amounts of maintenance data from organizational units. The military maintenance system is further subdivided into different work centers (or organizations) performing maintenance or repairs ranging from very specialized (for example, circuit card repair) to less specialized (general inspections or repairs). The organizational units are responsible to ensure a minimum number of aircraft are mission capable at all times - and thus utilize cannibalization practices when needed. Components may be removed from an aircraft and sent to a second level of maintenance for repair. The organizations are also supported by engineering and logistics functions in order to maintain readiness.

Maintenance data is typically analyzed by developing trend plots or regression analysis, which provide information for decision making. The data is challenging to analyze since the captured variables are both quantitative and qualitative. Additionally, variables may not exhibit correlation, and techniques such as regression may not work well. Finally, the data may contain complex underlying patterns which are not immediately obvious.

\section{Results and Discussion}

The case analysis included 51 unique aircraft with 690 documented discrepancies covering five unique system codes of radio, navigation, and electronic warfare systems. The remaining aircraft within the data set did not have any cannibalization actions associated with the aforementioned system codes. The data set incorporated 14 variables that included categorical and numerical features. The data set was selected from a larger database that contained the maintenance work orders for the EA-6B aircraft over several years. The maintenance data set included reported discrepancies (both reported by pilots and ground crew) organized by system (or work unit code). The data set included records associated with the cannibalization action on the donor aircraft and the aircraft that received the repair. Thus, each cannibalization action incurred two records (one for the donor and one for the receiver aircraft). The donor discrepancy was identified by a unique coding scheme already within the data set. As illustrated in Figure 3, whenever a repair is satisfied using a cannibalized part the corrective action contains "Cannibalized (BUNO: XXXXXX MCN: XXXXXXX)." 


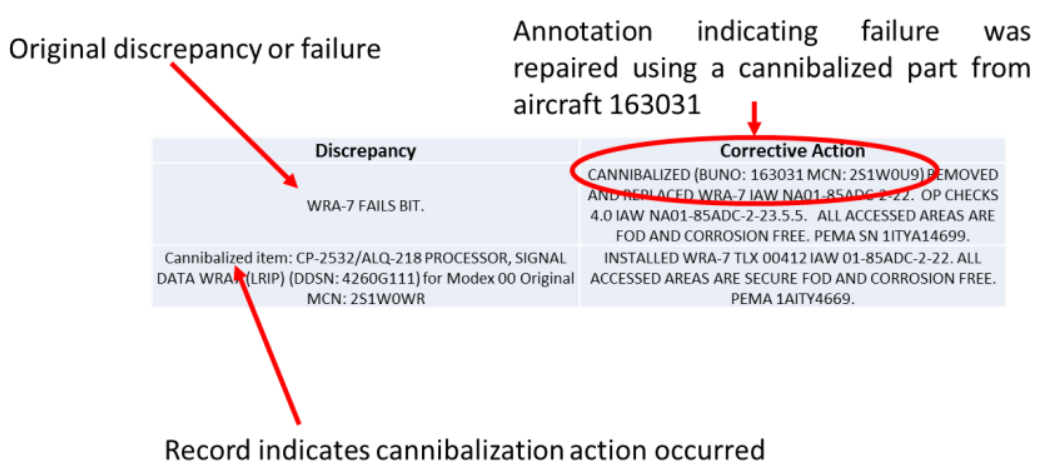

Figure 3. Coding of Cannibalization Actions

The numeric variables, particularly the partially mission capable hours, indicated large amounts of variation, and several potential outliers. Descriptive statistics are provided in Table 1 along with boxplots in Figure 4. Descriptive statistics indicated the quantitative variable distributions were unimodal, although highly positively skewed.

In order to determine if the outliers (based on the Interquartile Range) were valid or invalid, and to ensure sufficient data quality, all suspect records were manually assessed. Specifically, the review included assessing if any inconsistencies were found (for example, maintenance performed but no maintenance time logged) by subject matter experts familiar with Navy data collection procedures. Four records were flagged as invalid and removed from the data set. All zero delay times were reviewed for accuracy. Next, the data set was prepared for ingestion into a machine-learning algorithm by converting numerical variables to nominal scales of ZERO, LOW, MEDIUM, and HIGH based on their interquartile range.

Table 1.

Descriptive Statistics for Training Dataset (Zero Values Removed)

\begin{tabular}{|l|l|l|l|}
\hline Variable & Mean & Standard Deviation & IQR \\
\hline Elapsed Maintenance Time & 2.2 & 3.8 & 2.2 \\
\hline Awaiting Maintenance Time & 73.7 & 323.9 & 23.5 \\
\hline Awaiting Parts Time & 155.6 & 875.9 & 110.7 \\
\hline Non Mission Capable Time & 8280.0 & 13868.0 & 20216.0 \\
\hline Partially Mission Capable Time & 424.0 & 2273.0 & 132.0 \\
\hline
\end{tabular}




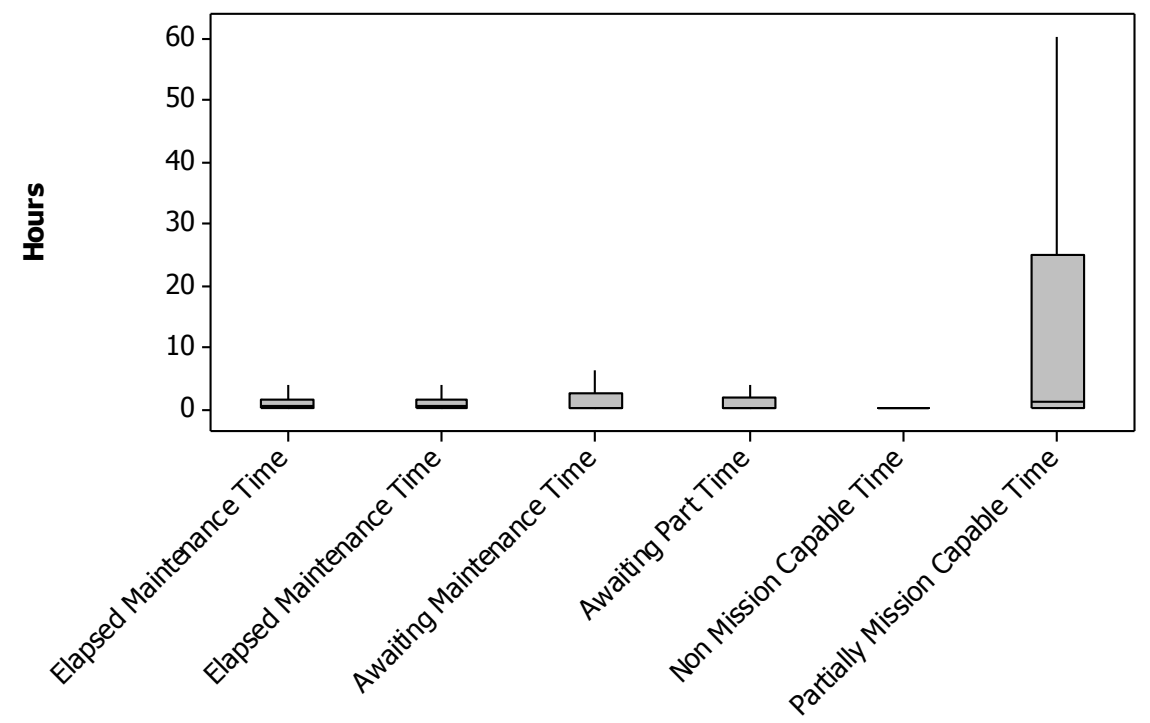

Figure 4. Boxplots for Case Analysis Dataset

Several categorical variables, such as the aircraft owner organizational code and organizational code of the repair personnel, were included in the data set. As illustrated in Figure 5, the frequency distribution of these categorical variables was diverse. Reduction of variable cardinality may have improved results; however, it was not further investigated in this study.

The potential linear correlation between variables were investigated using the Pearson correlation coefficient. The coefficients ranged from -0.001 to 0.120 , indicating that there was not a strong linear correlation between variables. A matrix plot was constructed to evaluate if a regression model could be established (linear or non-linear) for the data set. The regression analysis specifically considered predicting awaiting parts time, based on the assumption that awaiting parts may be indicative of requiring a cannibalization action (Okyere-Boateng, 2015; Peter Bogdanowicz, 2013). A regression model could not be established.

Tabu search was utilized in order to derive both the underlying Bayesian network structure and quantification of the associated conditional probability tables. The analysis considered the target feature (what we aim to predict) of receiving a cannibalized part, the owner organizational code, and action organization code. These variables were important from a practical implementation standpoint. The tabu algorithm utilized the Bayes local score metric and was limited to a maximum of two parents, 15 iterations, and a tabu list size of 10 due to computational burden. The obtained results are provided in Table 2. 


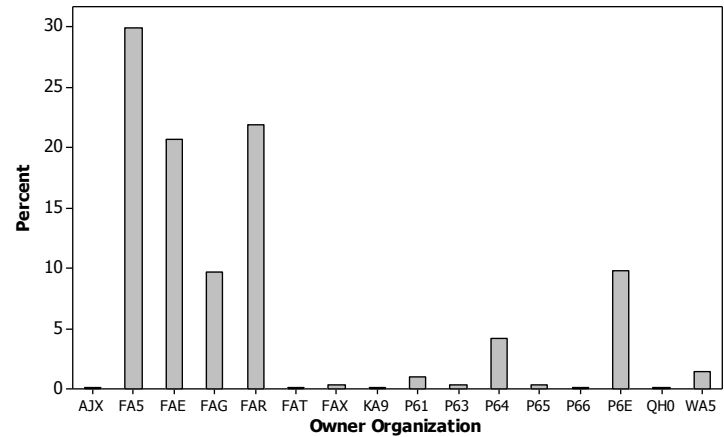

Percent within all data.

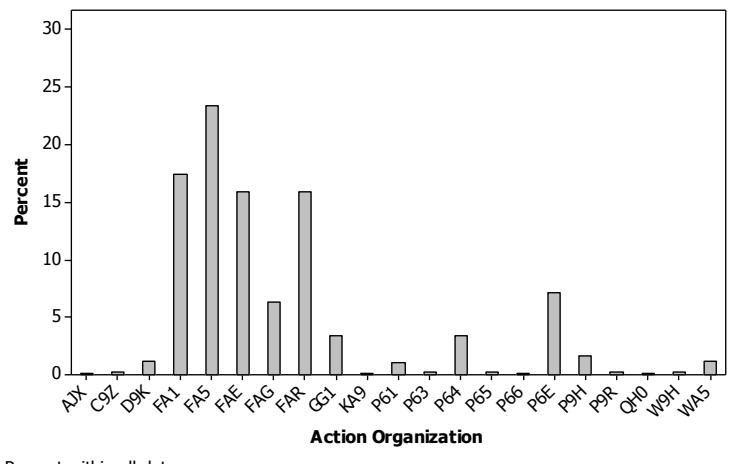

Percent within all data.

Figure 5. Owner and Action Organization Distribution

Table 2.

Machine Learning Algorithm Results

\begin{tabular}{|l|l|l|l|}
\hline Target Variable & $\begin{array}{l}\text { Average } \\
\text { Positive Rate (All } \\
\text { Classes) }\end{array}$ & $\begin{array}{l}\text { Average } \\
\text { Positive Rate (All } \\
\text { Classes) }\end{array}$ & $\begin{array}{l}\text { F- } \\
\text { measure }\end{array}$ \\
\hline $\begin{array}{l}\text { Received } \\
\text { Cannibalized Part }\end{array}$ & 86.9 & 54.7 & 86.7 \\
\hline $\begin{array}{l}\text { Owner } \\
\text { Organization }\end{array}$ & 87.5 & 3.3 & 87.0 \\
\hline Act Organization & 96.3 & 0.4 & 95.4 \\
\hline $\begin{array}{l}\text { Awaiting Parts } \\
\text { Hours }\end{array}$ & 73.6 & 8.0 & 74.2 \\
\hline
\end{tabular}


The high false alarm rate for the received cannibalized part was further investigated. Such a high false alarm rate was concerning and may erode the practical significance of the developed model. The variable could take on a value of either "yes" (part required cannibalization) or "no" (part satisfied through regular supply chain). The false alarm rate was only 7 percent when the class label was "yes." Thus, if the algorithm predicted that a cannibalization part is required, the false alarm rate was sufficiently low for practical significance. Prediction of the owner organization and action organization was deemed an important finding. The high significance when predicting these target variables will allow decision makers to quantify cannibalization risk to include potential maintenance requirements. The high F-measure scores (scale 0 to 100) indicated the algorithm achieved both high recall and precision in all cases. The derived Bayesian network is provided in Figure 6. The CPTs for variables with high cardinality are excluded in the graphic due to spacing limitations.

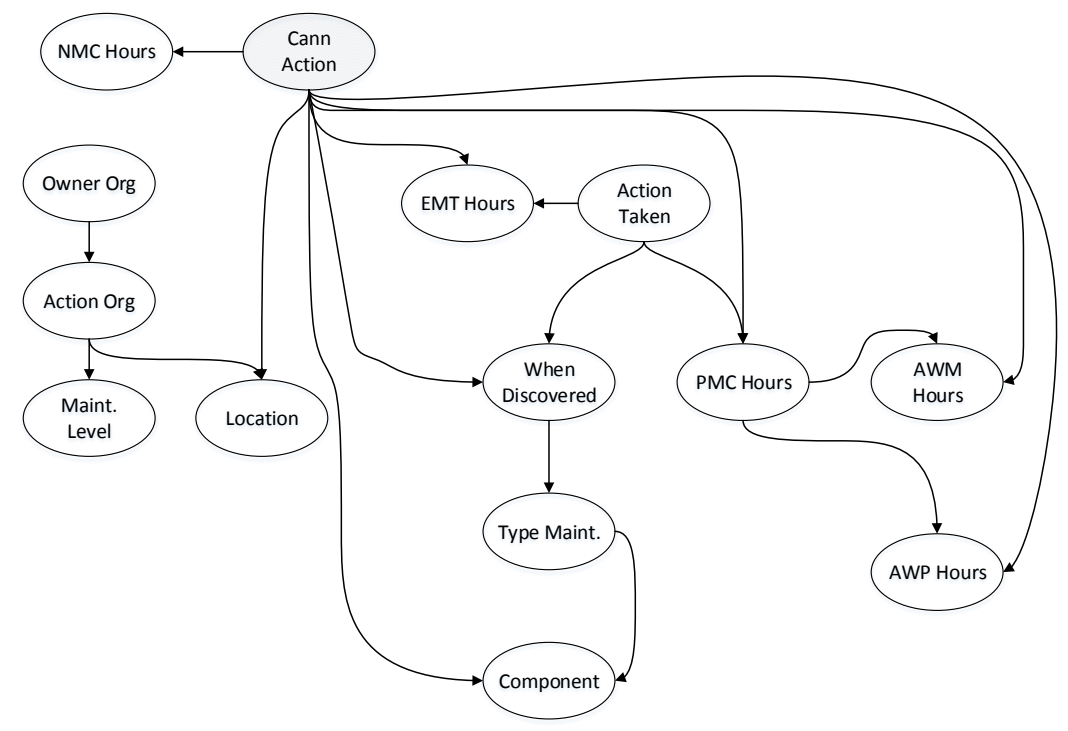

Figure 6. Derived Bayesian Network to predict cannibalization actions

\section{Conclusion, Future Research and Study Limitations}

The research indicated that it is feasible to apply Bayesian networks to predict aircraft cannibalization risk, given a real-world maintenance data set. The contribution fills an important gap within current literature, provides a 
methodology, and identifies a causal network that can inform decision making and several engineering processes to include reverse engineering.

There are several areas where the study can be expanded. First, the high false alarm rate should be investigated further. Variable reduction techniques, or reducing the cardinality and variation within the training set may reduce the rate. Additionally, a larger sample size may also improve the results.

Rhe derived causal network should be explored further. Variables such as the number of available spares, obsolescence, and the repair rate, may impact the number of cannibalizations. Additionally, variables describing the attributes of the maintenance personnel and organizations may also impact the results. The causal network should be evaluated by subject matter experts, possibly combined with a larger surveying of maintenance personnel to refine the model and incorporate variables not captured within the maintenance management system. The analysis should include a comparison of network structure changes if additional data is obtained. Research is also required in order to develop a user interface and decision support system utilizing the developed model. The framework must consider how knowledge is conveyed to the decision maker, re-validate the causal network as new data is integrated, and track algorithm performance versus readiness metrics. 


\section{References}

Albright, T. L., Gerber, C. A., \& Juras, P. (2014). How Naval aviation uses the balanced scorecard. Strategic Finance, 10, 21-28.

Bouckaert, R. R. (1995). Bayesian belief networks: From construction to inference. Utrecht: University of Utrecht.

Cheung, K. L., \& Hausman, W. H. (1995). Multiple failures in a multi-item spares inventory model. IIE Transactions, 27(2), 171-180.

Durupt, A., Sebastien, R., Ducellier, G., \& Bricogne, M. (2010). KBRE: A proposition of a reverse engineering process by a KBE system. International Journal for Interactive Design and Manufacturing, 4(4), 227-237.

Enslen, W. J. (2004). Situational cannibalization: a viable support alternative. Logistics Spectrum.

Fisher, W. W. (1986). The performance of cannibalization policies in a maintenance system with spares, repair, and resource constraints. Naval Research Logistics Quarterly, 33, 1-15.

Fisher, W. W. (1989). An improved simulation model for cannibilization policy performance comparisons in a complex maintenance system. Simulation, 52(4), 154-1648.

Government Accountability Office. (2001). Cannibalizations adversely affect personnel and maintenance. Washington D.C.: Government Accountability Office.

Griffin, J., \& Tomlinson, L. (2016, May 14). 'Wiped Out': Air Force losing pilots and planes to cuts, scrounging for spare parts. Fox News Politics. Retrieved from http://www.foxnews.com/politics/2016/05/14/wiped-out-air-force-losing-pilotsand-planes-to-cuts-scrounging-for-spare-parts.html

Kaiser, J. L., Bland, C. L., \& Klinke II, D. J. (2016). Identifying causal networks Linking cancer processes and anti-tumor immunity Using Bayesian network inference and metagene constructs. Biotechnology Progress, 32(2), 470-479.

Kellerher, J. D., Mac Namee, B., \& D'Arcy, A. (2015). Fundamentals of machine learning for predictive data analytics. Cambridge, MA: The MIT Press.

Li, X., Chen, G., \& Zhu, H. (2016). Quantitative risk analysis on leakage failure of submarine oil and gas pipelines using Bayesian network. Process Safety and Environmental Protection, 103, 163-173.

Liu, Y., \& Shen, J. (2015). Research on equipment predictive maintenance strategy based on big data technology. International Conference on Intelligent Transportation, Big Data \& Smart City. Halong Bay, Vietnam: IEEE. 
Margaritis, D. (2003). Learning Bayesian network model structure from data. Pittsburgh, PA: Carnegie Mellon University.

Okyere-Boateng, K. O. (2015). A Between-squadron analysis of cannibalization on the $M V$-22. Monterey, CA: Naval Post Graduate School.

Peter Bogdanowicz. (2013). Defense Technical Information Center (DTIC). Retrieved from http://www.dtic.mil/ndia/2013/system/WBogdanowicz.pdf

Rolfe, A. J. (1970). Markov chain analysis of a situation where cannibilization is the only repair activity. Washington, DC: RAND Corporation.

Salman, S., Cassady, C. R., Pohl, E. A., \& Ormon, S. W. (2007). Evaluating the impact of cannibalization on fleet performance. Quality and Reliability Engineering International, 23, 445-457.

Seck, H. H. (2016, 5 26). Eisenhower Strike Group to fight ISIS with cannibalized parts. Military.com. Retrieved from http://www.military.com/dailynews/2016/05/26/eisenhower-strike-group-to-fight-isis-with-cannibalizedparts.html

Sung, C., \& Kim, S. (2001). Analysis of a multi-part spares inventory system subject to ambiguous fault isolation. Journal of the Operational Research Society, 52, 418432.

Yang, S., Chang, \& K. (2002). Comparison of score metrics for Bayesian network learning. IEEE Transactions on Systems, Man, and Cybernetics - Part A: Systems and Humans, 32(3), 2002.

Zargoush, M., Alemi, F., Vinzi, V. E., Vang, J., \& Kheirbek, R. (2014). A psychological approach to learning causal networks. Health Care Management Science(17), 194-201.

Zhang, R., \& Ghanmi, A. (2014). Detailed cannibalization decision making for maintenance systems in the military context. 8th Annual IEEE Systems Conference (SysCon). Ottawa, ON, Canada. 